\title{
Antiferromagnetism and superconductivity of the two-dimensional extended $t-J$ model
}

\author{
C.T. Shih ${ }^{1}$, J.J. Wu ${ }^{1}$, Y.C. Chen ${ }^{1}$, C.Y. Mou ${ }^{2}$, C.P. Chou $^{2}$, R. Eder ${ }^{3}$, and T.K. Lee ${ }^{4}$ \\ ${ }^{1}$ Department of Physics, Tunghai University, Taichung, Taiwan \\ E-mail: ctshih@mail.thu.edu.tw \\ ${ }^{2}$ Department of Physics, National Tsing Hua University, Hsinchu, Taiwan \\ ${ }^{3}$ Institut für Festkörperphysik, Forschungszentrum Karlsruhe, Germany \\ ${ }^{4}$ Institute of Physics, Academia Sinica, Nankang, Taiwan
}

Received January 13, 2005

\begin{abstract}
The mechanism of high-temperature superconductivity (HTS) and the correlation between the antiferromagnetic long-range order (AFLRO) and superconductivity (SC) phases are the central issues of the study of HTS theory. SC and AFLRO of the hole-doped two-dimensional extended $t-J$ model are studied by the variational Monte Carlo method. The results show that SC is greatly enhanced by the long-range hopping terms $t^{\prime}$ and $t^{\prime \prime}$ for the optimal and overdoped cases. The phase of coexisting SC and AFM in the $t-J$ model disappears when $t^{\prime}$ and $t^{\prime \prime}$ are included. It is concluded that the extended $t-J$ model provides a more accurate description for HTS than the traditional $t-J$ model does. The momentum distribution function $n(\mathbf{k})$ and the shape of Fermi surface play critical roles for establishing the phase diagram of HTS materials.
\end{abstract}

PACS: 74.20.-z, 74.25.На, 71.18+y

\section{Introduction}

The two-dimensional (2D) $t-J$ model has been proposed to provide the mechanism of superconductivity (SC) $[1,2]$ right after the discovery of high-temperature superconductivity (HTS). This idea quickly gained momentum when variational calculations showed that the doping dependence of pairing correlation [3,4] and the phase diagram of the antiferranagnetic long-range order (AFLRO) and SC seem to agree with experimental results fairly well [5]. However, the calculation beyond variational method showed that SC of pure 2D $t-J$ model was not large enough to explain such high transition temperature of the cuprates [6]. Up to now, this issue is still to be settled [7-9].

Interplay between the $d$-wave SC and AFLRO is another one of the critical issues in the physics of HTS $[10,11]$. Early experimental results showed the existence of AFLRO at temperature lower than the Néel temperature $T_{N}$ in the insulating perovskite parent compounds of the cuprates. When charge carriers are doped, AFLRO is destroyed quickly and then SC appears. In most thermodynamic measurements for hole doped cuprates, AFLRO does not coexist with SC [12] and disappears completely around doping density $\delta_{h} \sim 5 \%$. However, recent experiments such as muon spin rotation and elastic neutron scattering show that the spin density wave (SDW) may compete, or coexist with SC [13-18]. These results suggest that AFLRO may coexist with SC but the possibility of inhomogeneous phases is not completely ruled out.

For the theoretical part of this issue, analytical and numerical studies of the $t-J$ model show that at half-filling, the $d$-wave resonating valence bond (RVB) state with AFLRO is a good trial wave function (TWF) and SC is absent due to the constraint of no-double-occupancy. Upon doping, the carriers become mobile and SC sets in while AFLRO is quickly suppressed. However, AFLRO will survive until the hole density $\delta_{h}>10 \%$, which is much larger than the critical density observed by experiments. SC and AFLRO coexist in the very underdoped regime [5,19-22].

The discrepancies imply that the $t-J$ model may be insufficient to describe the physics of HTS. On the 
other hand, there are several experimental and theoretical studies suggesting the presence of the next- and third-nearest-neighbor hopping terms $t^{\prime}$ and $t^{\prime \prime}$ in cuprates. For example, the topology of the large Fermi surface (FS) and the single-hole dispersion studied by angle-resolved photoemission spectroscopy (ARPES), and the asymmetry of phase diagrams of the electron- and hole-doped cuprates can be understood by introducing these terms [23].

It is suggested that the longer range hopping terms may play important roles on the mechanism of HTS. Results of band-structure calculations [24,25] and experimental analysis [26] show that $T_{C}$ is enhanced by the next-nearest neighbor hopping $t^{\prime} / t$, and the highest $T_{c \text {, max }}$ for different monolayer hole doped cuprates strongly correlates with $t^{\prime} / t$. However, this contradicts with previous results $[27,28]$ of exact calculations that for the hole doped systems, introducing $t^{\prime}$ into the $t-J$ model will suppress pairing.

We will discuss the model and the trial wave function in Sec. 2, and the variational Monte Carlo (VMC) method results for SC, AFLRO, and the shape of the Fermi surface in Sec. 3. At last we will make a summary in Sec. 4.

\section{The model and the wave functions}

The Hamiltonian of the extended $t-J$ model is

$$
\begin{aligned}
H=H_{t} & +H_{J}=-\sum_{i j} t_{i j}\left(\tilde{c}_{i, \sigma}^{\dagger} \tilde{c}_{j, \sigma}+\text { H.c. }\right)+ \\
& +J \sum_{\langle i, j\rangle}\left(\mathbf{S}_{i} \cdot \mathbf{S}_{j}-\frac{1}{4} n_{i} n_{j}\right)
\end{aligned}
$$

where $t_{i j}=t, t^{\prime}, t^{\prime \prime}$, and 0 for sites $i$ and $j$ are the nearest, next nearest, third nearest neighbors, and others, respectively. $\langle i, j\rangle$ in $H_{J}$ means the spin-spin interaction occurs only for nearest neighbors. $\tilde{c}_{i, \sigma}=$ $=\left(1-n_{i,-\sigma}\right) c_{i, \sigma}$, satisfies the no-double-occupancy constraint. At half-filling, the system is reduced to the Heisenberg Hamiltonian $H_{J}$. As carriers are doped into the parent compound, $H_{t}$ is included in the Hamiltonian.

To solve the ground state wave function of this Hamiltonian, three mean-field order parameters are introduced [21,29]: the staggered magnetization $m_{S}=\left\langle S_{A}^{z}\right\rangle=-\left\langle S_{B}^{z}\right\rangle$, where the lattice is divided into $A$ and $B$ sublattices, the uniform bond order parameters $\chi=\left\langle\sum_{\sigma} c_{i \sigma}^{\dagger} c_{j \sigma}\right\rangle$, and $d$-wave RVB $(d$-RVB) one $\Delta=\left\langle c_{j \downarrow} c_{i \uparrow}-c_{j \uparrow} c_{i \downarrow}\right\rangle$ if $i$ and $j$ are n.n. sites in the $x$ direction and $-\Delta$ for the $y$ direction. The Lee-Shih wave function (WF), which is the mean-field ground state $\mathrm{WF}$, is
$\left|\Psi_{L S}\right\rangle=P_{d}\left(\sum_{\mathbf{k} \in S B Z}\left(A_{\mathbf{k}} a_{\mathbf{k} \uparrow}^{\dagger} a_{-\mathbf{k} \downarrow}^{\dagger}+B_{\mathbf{k}} b_{\mathbf{k} \uparrow}^{\dagger} b_{-\mathbf{k} \downarrow}^{\dagger}\right)\right)^{N_{S} / 2}|0\rangle$,

where $N_{s}$ is the total number of sites and

$$
A_{\mathbf{k}}=\left(E_{\mathbf{k}}^{(1)}+\xi_{\mathbf{k}}^{-}\right) / \Delta_{\mathbf{k}}, \quad B_{\mathbf{k}}=-\left(E_{\mathbf{k}}^{(2)}-\xi_{\mathbf{k}}^{+}\right) / \Delta_{\mathbf{k}}
$$

with

$$
E_{\mathbf{k}}^{(1)}=\left(\xi_{\mathbf{k}}^{-2}+\Delta_{\mathbf{k}}^{2}\right)^{1 / 2}, \quad E_{\mathbf{k}}^{(2)}=\left(\xi_{\mathbf{k}}^{+2}+\Delta_{\mathbf{k}}^{2}\right)^{1 / 2} .
$$

Here $\Delta_{\mathbf{k}}=\frac{3}{4} J \Delta\left(\cos k_{x}-\cos k_{y}\right)$. Energy dispersions for the two SDW bands are

$$
\xi_{\mathbf{k}}^{ \pm}= \pm\left[\left(\varepsilon_{\mathbf{k}}+\mu\right)^{2}+\left(J m_{S}\right)^{2}\right]^{1 / 2}-\mu^{\prime}
$$

with

$$
\begin{gathered}
\varepsilon_{\mathbf{k}}=-2\left(t \delta+\frac{3}{8} J \chi\right)\left(\cos k_{x}+\cos k_{y}\right) . \\
a_{\mathbf{k} \sigma}=\alpha_{\mathbf{k}} c_{\mathbf{k} \sigma}+\sigma \beta \beta_{\mathbf{k}} c_{\mathbf{k}+\mathbf{Q} \sigma}
\end{gathered}
$$

and $b_{\mathbf{k} \sigma}=-\sigma \beta_{\mathbf{k}} c_{\mathbf{k} \sigma}+\alpha_{\mathbf{k}} c_{\mathbf{k}+\mathbf{Q} \sigma}$, where $\mathbf{Q}=(\pi, \pi)$,

$$
\begin{aligned}
& \alpha_{\mathbf{k}}^{2}=\frac{1}{2}\left\{1-\left[\left(\varepsilon_{\mathbf{k}}+\mu^{\prime}\right) /\left(\xi_{\mathbf{k}}^{+}+\mu^{\prime}\right)\right]\right\}, \\
& \beta_{\mathbf{k}}^{2}=\frac{1}{2}\left\{1+\left[\left(\varepsilon_{\mathbf{k}}+\mu^{\prime}\right) /\left(\xi_{\mathbf{k}}^{+}+\mu^{\prime}\right)\right]\right\},
\end{aligned}
$$

are the operators of the lower and upper SDW bands, respectively.

$$
\mu^{\prime}=\mu+4 t_{v}^{\prime} \cos k_{x} \cos k_{y}+2 t_{v}^{\prime \prime}\left(\cos 2 k_{x}+\cos 2 k_{y}\right),
$$

where $\mu$ is the chemical potential which determines the number of electrons, $t_{v}^{\prime}$ and $t_{v}^{\prime \prime}$ are variational parameters corresponding to the next and third nearest neighbor hoppings. $t_{v}^{\prime}$ and $t_{v}^{\prime \prime}$ are not necessarily equal to the bare values $t^{\prime}$ and $t^{\prime \prime}$ because the constraint strongly renormalizes the hopping amplitude. Note that the summation in Eq. (2) is taken over the sublattice Brillouin zone (SBZ). The operator $P_{d}$ enforces the constraint of no doubly occupied sites for cases with finite doping.

For the half filled case, $\mu=t^{\prime}=t^{\prime \prime}=0$ and the optimal variational energy of this trial wave function (TWF) obtained by tuning $\Delta$ and $m_{s}$ in the VMC simulation is $-0.332 J$ per bond which is within $1 \%$ of the best estimate of the ground state energy of the Heisenberg model [30]. For the case of pure AFLRO without $\Delta$, energy per bond is about 3 to $4 \%$ higher.

Upon doping, there are two methods to modify the TWF: one is to use a nonzero $\mu$ to control the filling of the SDW bands [29], the other is to create charge excitations from the half-filled ground states [31]. For 
the former method, the TWF is optimized by tuning $\Delta, m_{s}, t_{v}^{\prime}, t_{v}^{\prime \prime}$ and $\mu$. Note that for larger doping densities, AFLRO disappears $\left(m_{s}=0\right)$ and the WF reduces to the standard $d$-RVB WF. For the latter method, the WF is the «small Fermi pocket» state $\left|\Psi_{p}\right\rangle$ :

$\left|\Psi_{p}\right\rangle=P_{d}\left(\sum_{\mathbf{k} \in S B Z, \mathbf{k} \notin Q_{p}}\left(A_{\mathbf{k}} a_{\mathbf{k} \uparrow}^{\dagger} a_{-\mathbf{k} \downarrow}^{\dagger}+B_{\mathbf{k}} b_{\mathbf{k} \uparrow}^{\dagger} b_{-\mathbf{k} \downarrow}^{\dagger}\right)\right)^{N_{S} / 2}|0\rangle$.

The k-points in $Q_{p}$ are the momenta of the electron singlet pairs (with momenta and spin $(\mathbf{k} \uparrow,-\mathbf{k} \downarrow)$ ) removed from the half-filled FS. Thus the number of holes is twice of the number of k-points in $Q_{p}, \mu, t_{v}$ and $t_{v}^{\prime \prime}$ are identical to zero in Eq. (2) because the size and shape of FS are determined by the choice of $Q_{p}$. Note that no matter what k's are chosen in $Q_{p}$, the total momentum of the WF is zero. k's can be viewed as «hidden quantum numbers» of the wave function.

In general, for the ground state the set $Q_{p}$ should be determined variationally. As we expected, it agrees well with the rigid band picture for very underdoped systems [31]. For example, there is only one point in the two-hole system.

The variational energies for several choices of $\mathbf{k}$ in a $12 \times 12$ lattice are shown in Fig. 1 . It can be seen that for both $\left(t^{\prime}, t^{\prime \prime}\right)=(-0.1,0.05) t$ (full circles) $\left(t^{\prime}, t^{\prime \prime}\right)=$ $=(-0.3,0.2) t$ (open circles) cases, the $\mathbf{k}$ with lowest energy is $(\pi / 2, \pi / 2)$. The $\mathbf{k}$ 's with the second lowest energy are $(2 \pi / 3, \pi / 3)$ and $(\pi / 2, \pi / 3)$ for $\left(t^{\prime}, t^{\prime \prime}\right)=$ $=(-0.1,0.05) t$ and $\left(t^{\prime}, t^{\prime \prime}\right)=(-0.3,0.2) t$, respectively.

According to the rigid-band assumption, we expect that the best choice of $Q_{p}$ for the 4-hole system is

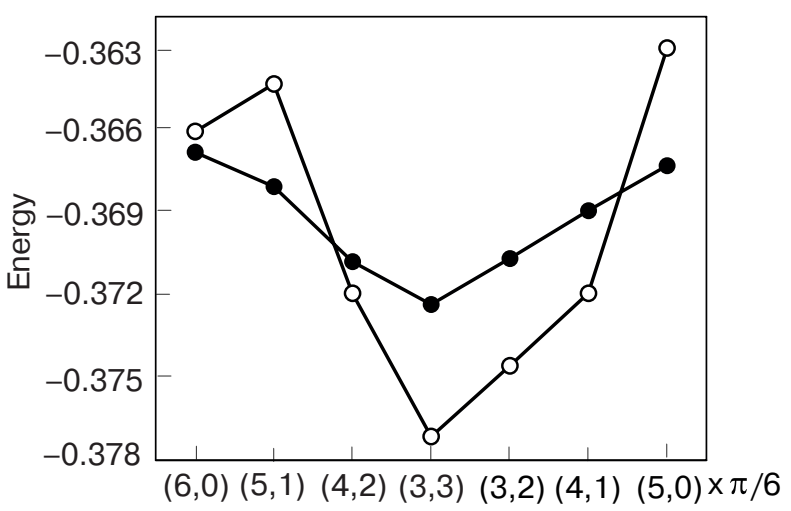

Fig. 1. Energies for two holes in a $12 \times 12$ lattice for $J / t=0.3, \quad\left(t^{\prime}, t^{\prime \prime}\right)=(-0.1,0.05) t \quad$ (full circles) and $\left(t^{\prime}, t^{\prime \prime}\right)=$ $=(-0.3,0.2) t$ (open circles), respectively. $\mathbf{k}$ is the «hidden quantum number» corresponding to the momentum of the pair removed from the half filled Fermi surface. Note that the total momenta of all the wave functions are zero. $\{(\pi / 2, \pi / 2),(-\pi / 2, \pi / 2)\}$. And $Q_{p}$ 's for the 6-hole system with

$$
\left(t^{\prime}, t^{\prime \prime}\right)=(-0.3,0.2) t \text { and }\left(t^{\prime}, t^{\prime \prime}\right)=(-0.1,0.05) t
$$

are

$$
\{(\pi / 2, \pi / 2),(-\pi / 2, \pi / 2),(\pi / 2, \pi / 3)\},
$$

and

$$
\{(\pi / 2, \pi / 2),(-\pi / 2, \pi / 2),(2 \pi / 3, \pi / 3)\},
$$

respectively.

Figure 2 shows the choices of $Q_{p}$ 's for several doping densities $(0 \sim 10$ holes $)$ for the $\left(t^{\prime}, t^{\prime \prime}\right)=(-0.3,0.2) t$ case. The validity of the rigid-band picture has been checked by comparing several $Q_{p}$ 's for the same number of holes for these very underdoped cases.

Another issue is that the choice of $Q_{p}$ may change the total symmetry of the WF. For example, Fig. 2,e shows that $Q_{p}$ for 8 holes is

$\{(\pi / 2, \pi / 2),(-\pi / 2, \pi / 2),(\pi / 2, \pi / 3),(-\pi / 2,-\pi / 3)\}$.

We can also choose

$$
\begin{gathered}
Q_{p}=\{(\pi / 2, \pi / 2),(-\pi / 2, \pi / 2), \\
(\pi / 2, \pi / 3),(\pi / 3, \pi / 2)\} .
\end{gathered}
$$
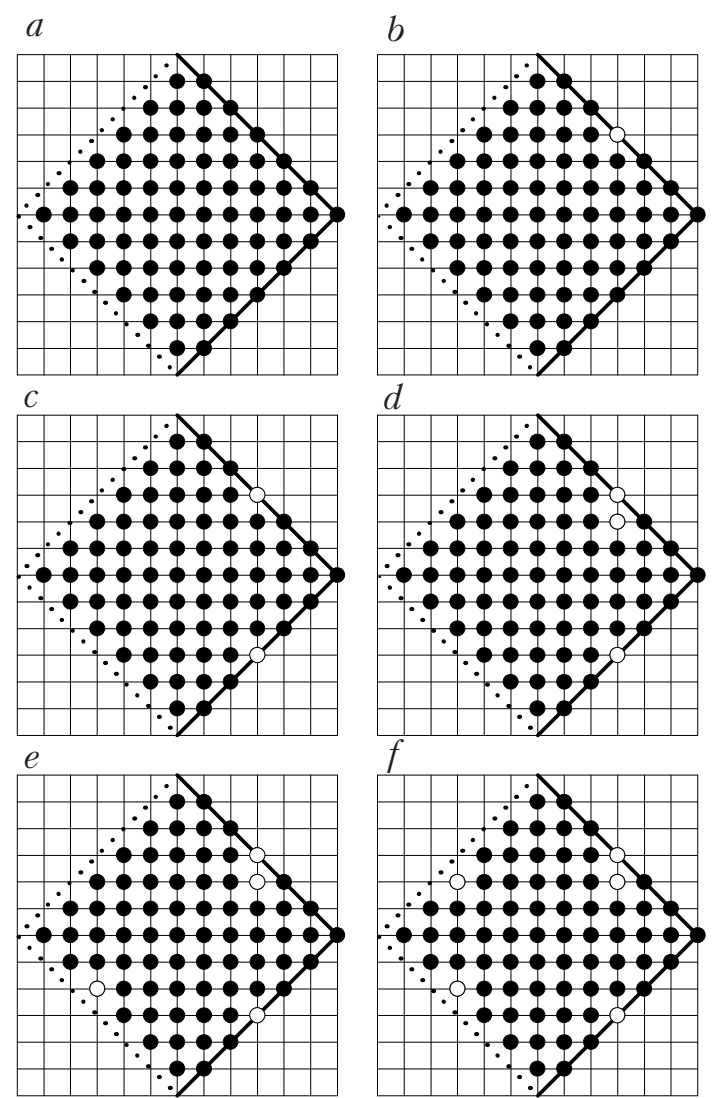

Fig. 2. Choices of $Q_{p}$ (open circles) for several doping densities for $t^{\prime} / t=-0.3$ and $t^{\prime \prime} / t=0.2$ in the $\mathbf{k}$-space. Full circles are the occupied k-points: $0(a), 2(b), 4(c), 6(d)$, $8(e), 10(f)$ holes, respectively. 
The variational energies of these two wave functions, long-range pair-pair correlation, and staggered magetization are almost identical (within error bars). Since $\mathbf{k}=( \pm \pi / 2, \pm \pi / 3)$ and $( \pm \pi / 3, \pm \pi / 2)$ are all degenerate for the two-hole system, the WF could also be degenerated for those $Q_{p}$ 's with k-points $(\pi / 2, \pm \pi / 2)$ and any two of $\mathbf{k}=( \pm \pi / 2, \pm \pi / 3)$ and $( \pm \pi / 3, \pm \pi / 2)$ for the 8-hole system. This conjecture has been verified numerically. Thus the best TWF should be a linear combination of all these WF's. For simplicity, we choose only one of the $Q_{p}$ in the following calculation. The properties of SC and AFLRO are not affected by this simplification [32].

\section{Results and discussion}

The staggered magnetization

$$
\langle M\rangle=\frac{1}{N_{s}}\left\langle\sum_{j} \mathrm{e}^{i \mathbf{Q} \cdot \mathbf{R}_{j}} S_{\mathbf{R}_{j}}^{z}\right\rangle,
$$

the momentum distribution function

$$
n(\mathbf{k})=\frac{1}{N_{s}} \sum_{i j \sigma} \mathrm{e}^{i \mathbf{k} \cdot\left(\mathbf{R}_{i}-\mathbf{R}_{j}\right)}\left\langle c_{i \sigma}^{\dagger} c_{j \sigma}\right\rangle,
$$

and the $d$-wave pair-pair correlation

$$
P_{d}(\mathbf{R})=\frac{1}{N_{s}}\left\langle\sum_{i} \Delta_{\mathbf{R}_{i}}^{\dagger} \Delta_{\mathbf{R}_{i}+\mathbf{R}}\right\rangle,
$$

where

$$
\Delta_{\mathbf{R}_{i}}=c_{\mathbf{R}_{i} \uparrow}\left(c_{\mathbf{R}_{i}+\hat{\mathbf{x}} \downarrow}+c_{\mathbf{R}_{i}-\hat{\mathbf{x}} \downarrow}-c_{\mathbf{R}_{i}+\hat{\mathbf{y}} \downarrow}-c_{\mathbf{R}_{i}-\hat{\mathbf{y}} \downarrow}\right)
$$
are measured for $J / t=0.3$ and $(a) t^{\prime}=t^{\prime \prime}=0$, (b) $\left(t^{\prime}, t^{\prime \prime}\right)=(-0.3,0.2) t$ and $(c)\left(t^{\prime}, t^{\prime \prime}\right)=(-0.1,0.05) t$ cases for the $12 \times 12$ lattice with periodic boundary condition. $P_{d}^{\text {ave }}$ is the averaged value of the long-range part $(|\mathbf{R}|>2)$ of $P_{d}(\mathbf{R})$. The optimal WF for different densities are determined by minimizing the variational energies among $\left|\Psi_{p}\left(m_{s}, \Delta,\left\{Q_{p}\right\}\right)\right\rangle$ and $\left|\Psi_{L S}\left(m_{s}, \Delta, t^{\prime}{ }_{v}, t_{v}^{\prime \prime}, \mu\right)\right\rangle$. We will discuss the results for these three cases in this section.

$$
\text { 3.1. } t^{\prime}=t^{\prime \prime}=0
$$

It can be seen in Fig. 3, $a$ that in the underdoped region for the $J / t=0.3, t^{\prime}=t^{\prime \prime}=0$ case, AFLRO coexists with SC for density smaller than $\delta_{c} \sim 10 \%$. The $\delta_{c}$ is smaller than the weak-coupling mean-field result $\sim 15 \%$ [21], but is still larger than the phase boundary of AFLRO determined by experiments $\left(\delta_{c}<5 \%\right)$. The energies of $\left|\Psi_{L S}\right\rangle$ are lower than those of $\left|\Psi_{P}\right\rangle$ for all doping densities in this case. This result is also consistent with the results reported by Himeda and Ogata [22]. Comparison of the VMC result with that of the weak-coupling one seems to indicate that the rigorous
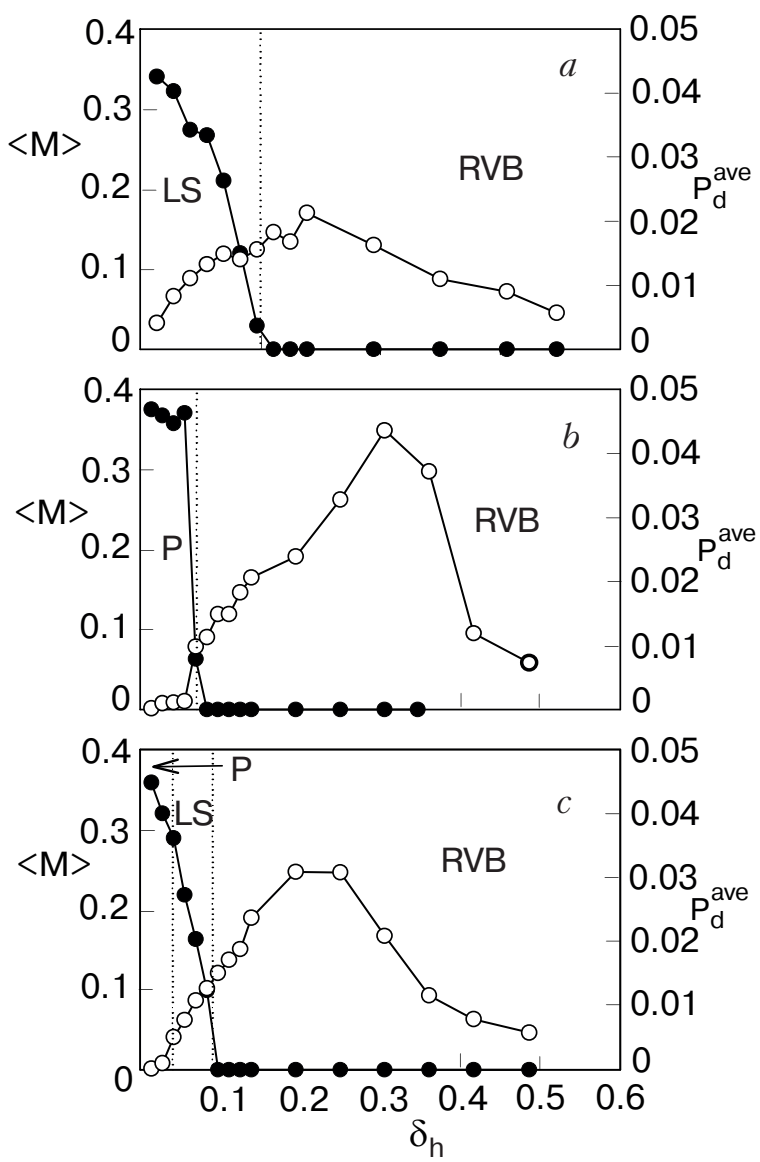

Fig. 3. $\langle M\rangle$ (full circles) and $P_{d}^{\text {ave }}$ (empty circles) for $J / t=0.3: \quad t^{\prime}=t^{\prime \prime}=0 \quad(a), \quad t^{\prime}=t^{\prime \prime}=(-0.3,0.2) t \quad(b), \quad$ and $\left(t^{\prime}, t^{\prime \prime}\right)=(-0.1,0.05) t(c)$ for hole doped $12 \times 12$ lattice. The vertical dash lines show the critical doping densities of level crossing. P $\left(\left|\Psi_{P}^{\prime}\right\rangle\right), \quad \operatorname{LS}\left(\left|\Psi_{L S}\right\rangle\right)$, and $\operatorname{RVB}\left(\left|\Psi_{L S}^{\prime}\right\rangle\right.$ with $\left.m_{S}=0\right)$ represent the best TWF's of each region.

no-double-occupancy constraint suppresses the AFLRO faster than the constraint-relaxed mean-field approximation.

$P_{d}^{\text {ave }}$ shows a dome-like shape which agrees well with the experiments except in the slightly doped AFLRO region. It is well known that the variational method usually overestimates the order parameters. Our previous studies using calculations beyond VMC show that $P_{d}^{\text {ave }}$ will be suppressed greatly when the $\mathrm{WF}$ is projected to the true ground state. Note that the two-hole binding energy becomes positive (no binding) in the thermodynamic limit [6].

$$
\text { 3.2. } t^{\prime} / t=-0.3 \text { and } t^{\prime \prime} / t=0.2
$$

Now we examine the phase diagram for $J / t=0.3$, $t^{\prime} / t=-0.3$ and $t^{\prime \prime} / t=0.2$, parameters for $\mathrm{YBCO}$ and BSCO compounds. The results are shown in Fig. $3, b$. It was found that level crossing occurs at $\delta_{c} \sim 0.06$. For $\delta_{h}<0.06,\left|\Psi_{p}\right\rangle$ is the ground state $\mathrm{WF}$ and $\langle M\rangle$ is a little larger than in the $t^{\prime}=t^{\prime \prime}=0$ case while $P_{d}^{\text {ave }}$ is suppressed by one order of magnitude. Thus there is 
AFLRO but no SC in this regime. For $\delta_{h}$ larger than 0.06 , the RVB state $\left(m_{S}=0\right.$ in $\left.\left|\Psi_{L S}\right\rangle\right)$ optimizes the energy. $P_{d}^{\text {ave }}$ increases and $\langle M\rangle$ drops to zero sharply. Unlike the $t^{\prime}=t^{\prime \prime}=0$ case, there is no region optimized by $\left|\Psi_{L S}\right\rangle$ with non-zero $m_{S}$. In conclusion, there is no coexistence of AFLRO and SC for the $\left(t^{\prime}, t^{\prime \prime}\right)=$ $=(-0.3,0.2) t$ case.

To show $\left|\Psi_{L S}\right\rangle$ and $\left|\Psi_{P}\right\rangle$ belong to two different types of WF, we calculate the overlap of them. $\left(\left\langle\Psi_{L S} \mid \Psi_{P}\right\rangle /\left(\left|\Psi_{L S} \| \Psi_{P}\right|\right)\right)$ is only 0.0113(4) [33]. The almost orthogonality of the two wave functions implies that the ground state WF's switch at the critical density.

The result that the critical $\delta_{h}$ for negative $t^{\prime} / t$ is smaller than that of $t^{\prime}=0$ case is consistent with the results evaluated by exact diagonalization $[35,36]$ and the suppression of coexistence of AFLRO and SC is consistent with the slave-boson mean-field theory [37].

For a little larger doping density $0.06<\delta_{h}<0.15$, it can be seen that $P_{d}^{\text {ave }}$ starts to grow but is still smaller in comparison with that in the $t^{\prime}=t^{\prime \prime}=0$ case. The suppression of $P_{d}^{\text {ave }}$ by $t^{\prime}$ and $t^{\prime \prime}$ in the underdoped regime is consistent with the results $[27,28]$ obtained the density matrix renormalization group (DMRG) method. Interestingly, for even larger $\delta_{h}, P_{d}^{\text {ave }}$ grows greatly and reaches the maximum at $\delta_{h} \sim 30 \%$ and the SC region extends to $\delta_{h} \sim 0.4$. The maximal $P_{d}^{\text {ave }}$ is larger than the $t^{\prime}=t^{\prime \prime}=0$ value at the same density by almost one order of magnitude, and about 2.5 times larger than the maximum of the optimal value of the $t^{\prime}=t^{\prime \prime}=0$ case. The enhancement of $P_{d}^{\text {ave }}$ may come from the deformation of the Fermi surface. The electron occupation at the $\mathbf{k}$-points near $(\pi, 0)$ is increased by a negative $t^{\prime}$. The results from exact diagonalization and slave-boson mean-field theory also show similar behavior [34].

The great enhancement of pairing due to $t^{\prime}$ may provide a possible mechanism for HTS. But the doping density $\delta_{\max }$ with maximal $P_{d}^{\text {ave }}$ is too large $(\sim 30 \%)$ in comparison with experiments $(15 \%)$. This discrepancy may disappear for the real ground state of the extended $t-J$ model. From our experience, if we do the calculation beyond VMC, the amplitude of $P_{d}^{\text {ave }}$ will be suppressed and $\delta_{\max }$ will move to a smaller value [6]. If this trend is true for the $t-J$ type models, we expect that $\delta_{\max }$ may move toward the more physical value. This conjecture will be investigated in the future.

\section{3. $t^{\prime} / t=-0.1$ and $t^{\prime \prime} / t=0.05$}

For the lanthanum materials with $t^{\prime} / t=-0.1$ and $t^{\prime \prime} / t=0.05$, the behaviors are more complex. It can be seen from Fig. 3,c that for the hole density $\delta_{h}<4 \%$ $\left|\Psi_{P}\right\rangle$ optimizes the variational energy and the phase in this region is ARFLO but no SC. For $4 \%<\delta_{h}<10 \%$,
$\left|\Psi_{L S}\right\rangle$ is the best TWF with nonzero $m_{s}$ and $\Delta$. AFLRO and SC coexist in the ground state of this density interval. For even larger dopings, $m_{s}$ in $\left|\Psi_{L S}\right\rangle$ vanishes and the phase becomes pure SC. The maximum of the SC dome is at $\delta_{h} \sim 20 \%$, and maximal $P_{d}^{\text {ave }}$ is about 1.5 times larger than the $t^{\prime}=t^{\prime \prime}=0$ value.

Since the phase transition comes from the level crossing of the two classes of states $\left|\Psi_{P}\right\rangle$ and $\left|\Psi_{L S}\right\rangle$, it is a first order phase transition. It is quite natural to have inhomogeneity in the system near the critical point [38]. It may also lead to other more novel inhomogeneous states such as stripe phase [39]. Another interesting result of our study is that the non-coexistence of SC and AFLRO is much more robust for systems with larger values of $t^{\prime} / t$ and $t^{\prime \prime} / t$ such as YBCO and BSCO [25]. For LSCO where $t^{\prime} / t$ and $t^{\prime \prime} / t$ are smaller, the tendency toward coexistence is larger and the possibility of inhomogeneous phase will become much more likely.

\subsection{Shape of the Fermi surface}

Figure 4 shows FS of both under- and overdoped systems with the parameter sets we discussed above. For the underdoped systems $\left(\delta_{h}=6 / 144\right)$, there is a large FS for the $t^{\prime}=t^{\prime \prime}=0$ case (Fig. 4,a) whereas a clear «Fermi pocket» for the $t^{\prime} / t=-0.3$ and $t^{\prime \prime} / t=0.2$ case (Fig. $4, b)$, whose ground state $\mathrm{WF}$ is $\left|\Psi_{P}\right\rangle$. The shape of the FS for $t^{\prime} / t=-0.1$ and $t^{\prime \prime} / t=0.05$ (Fig. $4, c)$ is placed between the previous two cases. The ground state WF is $\left|\Psi_{L S}\right\rangle$ but the pocket-like feature is still obvious. Being lack of a large FS is one of the possible reasons for the suppression of $P_{d}^{\text {ave }}$ by $t^{\prime}$ in the underdoped region.

For the overdoped systems $\left(\delta_{h}=44 / 144\right)$, the ground state WF for all the three cases are $\left|\Psi_{L S}\right\rangle$. They all have large FS's but with different shapes mainly determined by the parameters $t_{v}^{\prime}$ and $t_{v}^{\prime \prime}$. It is clear that the distortion of FS makes $n(\mathbf{k}=(\pi, 0))$ for the $t^{\prime} / t=-0.3$ and $t^{\prime \prime} / t=0.2$ (Fig. 4,e) case much larger than the other two. The shapes of FS for $t^{\prime}=t^{\prime \prime}=0$ (Fig. 4, $d$ ) and $t^{\prime} / t=-0.1$ and $t^{\prime \prime} / t=0.05$ (Fig. 4, $f$ ) are similar and the occupations near $(\pi, 0)$ are both small. For the $d$-wave SC, the electron pairs with momenta near $(\pi, 0)$ contribute to SC most. Thus $P_{d}^{\text {ave }}$ for $t^{\prime} / t=-0.3$ and $t^{\prime \prime} / t=0.2$ case is much larger than the other two.

Our results show that $P_{d}^{\text {ave }}$ is closely correlated with $n(\mathbf{k})$ and thus with the shape of FS. Figure 5 plots the maximal possible value of $P_{d}^{\text {ave }}$ for all doping densities as a function of $t^{\prime}$. The maximal $P_{d}^{\text {ave }}$ is proportional to $t^{\prime}$ in the range $0 \geq t^{\prime} \geq-0.3 \sim-0.4$. Beyond these values pairing is no longer enhanced. Coincidentally these values are about the same value of $t^{\prime} / t$ for mercury cuprates as estimated by Pavarini et al. [25] but much 

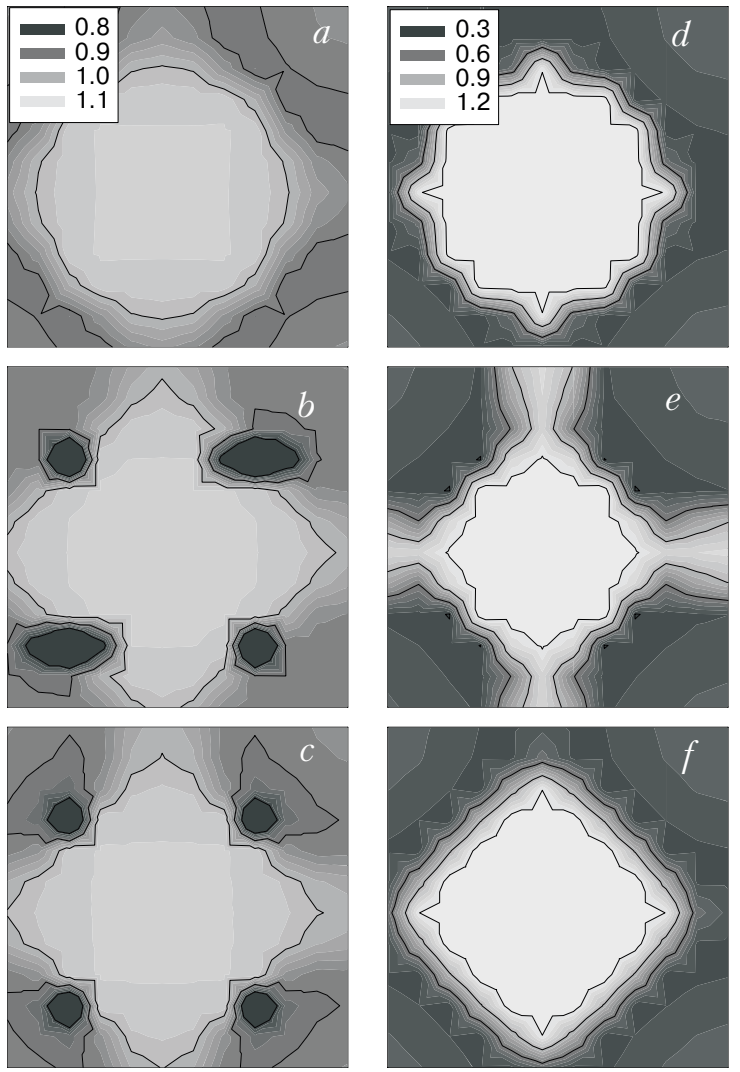

Fig. 4. Contour maps of $n(\mathbf{k}): t^{\prime}=t^{\prime \prime}=0(a),(d) ;\left(t^{\prime}, t^{\prime \prime}\right)=$ $=(-0.3,0.2) t(b),(e) ;\left(t^{\prime}, t^{\prime \prime}\right)=(-0.1,0.05) t(c),(f)$. The hole densities are $6 / 144$ for $(a),(b),(c)$, and $44 / 144$ for $(d)$, $(e),(f)$, respectively.

larger than those reported in Ref. 24. Among all the cuprate series, mercury cuprate maintains the record of having highest $T_{C}$ for almost a decade.

The decrease of $P_{d}^{\text {ave }}$ for $-t^{\prime} \geq 0.4$ in the overdoped regime such as $\delta=0.31$ is also likely the consequence of the change of the FS. $n(\mathbf{k}=(\pi, 0))$ is almost saturated at $-t^{\prime}=0.4$ and remains unchanged for larger $-t^{\prime}$. It is not difficult to recognize that as $-t^{\prime}$ becomes much larger than $t$, electrons will occupy separate regions around $\boldsymbol{k}=( \pm \pi, 0)$ and $\mathbf{k}=(0, \pm \pi)$. Hence the FS becomes disjoint pieces. Although at $-t^{\prime} / t=0.4$ the FS is still connected but this tendency is already observed. The density of states starts to decrease and this is probably the reason for the suppression of pairing beyond $-t^{\prime} / t^{\prime} \geq 0.4$.

\section{Summary}

In summary, a new $\mathrm{WF}\left|\Psi_{P}\right\rangle$ is proposed for the extended $t-J$ model for very low hole densities. The size and shape of the FS and of $\left|\Psi_{P}\right\rangle$ are determined by the choice of pairs with the momenta k's $\in Q_{p}$ removed from the half-filled system. The chosen k's are around the $(\pi / 2, \pi / 2)$ region in the $\mathbf{k}$-space for hole-doped materials. The behavior of $\left|\Psi_{P}\right\rangle$ is very different from

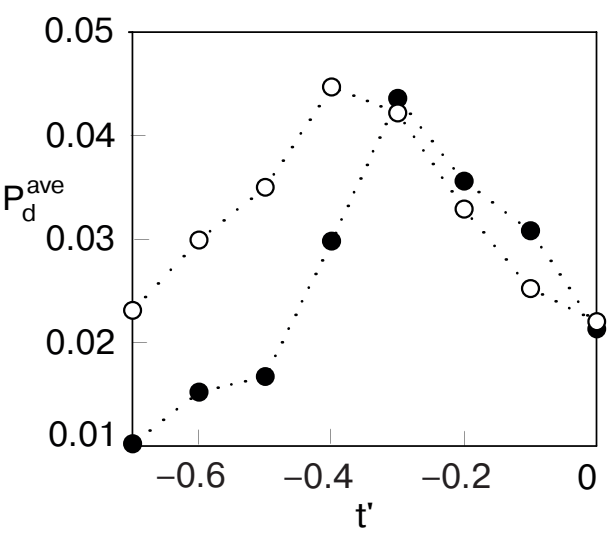

Fig. 5. Maximal $P_{d}^{\text {ave }}$ for different $t^{\prime}$ with $t^{\prime \prime}=-t^{\prime} / 2$ for $8 \times 8$ (open circle) and $12 \times 12$ (full circle) lattices.

that of $\left|\Psi_{L S}\right\rangle$ which optimizes the energy for the $t-J$ model. In contrast to $\left|\Psi_{P}\right\rangle$, the FS for the states of $\left|\Psi_{L S}\right\rangle$ is controlled by the chemical potential $\mu$ and the effective long-range hopping terms $t_{v}^{\prime}$ and $t_{v}^{\prime \prime}$.

There are three remarkable effects of $t^{\prime}$ and $t^{\prime \prime}$ for the extended $t-J$ model. First, the critical density where AFLRO vanishes is moved to more physical values. Second, the phase of coexisting AFLRO and SC is suppressed. If $t^{\prime}$ and $t^{\prime \prime}$ are large enough (corresponding to the YBCO or BSCO materials), the coexisting phase will disappear. Third, $P_{d}^{\text {ave }}$ is enhanced for the optimal and overdoped region, and suppressed for the underdoped region. This solves the controversy between the DMRG and band structure calculation results. The enhancement of $P_{d}^{\text {ave }}$ can be explained by the electron occupation near $(\pi, 0)$ and FS. These results offer a possible mechanism for HTS.

The work is supported by the National Science Council in Taiwan with Grant Nos. NSC-93-2112M-029-001-, 93-2112-M-007-009-, 93-2112-M-001-018-, and 93-2112-M029-009. Part of the calculations are performed in the IBM P690 and PC clusters in the Nation Center for High-performance Computing in Taiwan, and the PC clusters of the Department of Physics and Department of Computer Science and Engineering of Tunghai University, Taiwan. We are grateful for their help.

1. P.W. Anderson, Science 235, 1196 (1987).

2. F.C. Zhang and T.M. Rice, Phys. Rev. B37, 3759 (1988).

3. C. Gros, Phys. Rev. B38, 1196 (1988).

4. F.C. Zhang, C. Gros, T.M. Rice, and H. Shiba, Supercon. Sci. Tech. 1, 36 (1988).

5. T.K. Lee and S.P. Feng, Phys. Rev. B38, 11809 (1988).

6. C.T. Shih, Y.C. Chen, H.Q. Lin, and T.K. Lee, Phys. Rev. Lett. 81, 1294 (1998). 
7. S. Sorella, G.B. Martins, F. Becca, C. Gazza, L. Capriotti, A. Parola, and E. Dagotto, Phys. Rev. Lett. 88, 117002 (2002).

8. T.K. Lee, C.T. Shih, Y.C. Chen, and H.Q. Lin, Phys. Rev. Lett. 89, 279702 (2002).

9. S. Sorella, A. Parola, F. Becca, L. Capriotti, C. Gazza, E. Dagotto, and G. Martins, Phys. Rev. Lett. 89, 279703 (2002).

10. P.W. Anderson, The Theory of Superconductivity in the High- $T_{C}$ Cuprates, Princeton University Press, Princeton, NY (1997).

11. S.C. Zhang, Science 275, 1089 (1997).

12. C.M.A. Kastner, R.J. Birgeneau, G. Shirane, and Y. Endoh, Rev. Mod. Phys. 70, 897 (1998).

13. B. Lake, H.M. Rønnow, N.B. Christensen, G. Aeppli, K. Lefmann, D.F. McMorrow, P. Vorderwisch, P. Smeibidl, N. Mangkorntong, T. Sasagawa, M. Nohara, H. Takagi, and T.E. Mason, Nature 415, 299 (2002).

14. R.I. Miller, R.F. Kiefl, J.H. Brewer, J.E. Sonier, J. Chakhalian, S. Dunsiger, G.D. Morris, A.N. Price, D.A. Bonn, W.H. Hardy, and R. Liang, Phys. Rev. Lett. 88, 137002 (2002).

15. J.E. Sonier, K.F. Poon, G.M. Luke, P. Kyriakou, R.I. Miller, R. Liang, C.R. Wiebe, P. Fournier, and R.L. Greene, Phys. Rev. Lett. 91, 147002 (2003).

16. Y. Sidis, C. Ulrich, P. Bourges, C. Bernhard, C. Niedermayer, L.P. Regnault, N.H. Andersen, and B. Keimer, Phys. Rev. Lett. 86, 4100 (2001).

17. J.A. Hodges, Y. Sidis, P. Bourges, I. Mirebeau, M. Hennion, and X. Chaud, Phys. Rev. B66, 020501(R) (2002).

18. H.A. Mook, P. Dai, S.M. Hayden, A. Hiess, J.W. Lynn, S.-H Lee, and F. Doğan, Phys. Rev. B66, 144513 (2002).

19. G.J. Chen, R. Joynt, F.C. Zhang, and C. Gros, Phys. Rev. B42, R2662 (1990).

20. T. Giamarchi and C. Lhuillier, Phys. Rev. B43, 12943 (1991).

21. M. Inaba, H. Matsukawa, M. Saitoh, and H. Fukuyama, Physica C257, 299 (1996).
22. A. Himeda and M. Ogata, Phys. Rev. B60, R9935 (1999).

23. A. Damascelli, Z. Hussain, and Z.-X. Shen, Rev. Mod. Phys. 75, 473 (2003).

24. R. Raimondi, J.H. Jefferson, and L.F. Feiner, Phys. Rev. B53, 8774 (1996); L.F. Feiner, J.H. Jefferson and R. Raimondi, Phys. Rev. Lett. 76, 4939 (1996).

25. E. Pavarini, I. Dasgupta, T. Saha-Dasgupta, O. Jepsen, and O.K. Andersen, Phys. Rev. Lett. 87, 047003 (2001).

26. K. Tanaka, T. Yoshida, A. Fujimori, D.H. Lu, Z.-X. Shen, X.-J. Zhou, H. Eisaki, Z. Hussain, S. Uchida, Y. Aiura, K. Ono, T. Sugaya, T. Mizuno, and I. Terasaki, Phys. Rev. B70, 092503 (2004).

27. S.R. White and D.J. Scalapino, Phys. Rev. B60, R753 (1999)

28. G.B. Martins, J.C. Xavier, L. Arrachea, and E. Dagotto, Phys. Rev. B64, 180513 (2001).

29. T.K. Lee, and C.T. Shih, Phys. Rev. B55, 5983 (1997).

30. S. Liang, B. Doucot, and P.W. Anderson, Phys. Rev. Lett. 61, 365 (1988).

31. T.K. Lee, C.M. Ho, and N. Nagaosa, Phys. Rev. Lett. 90, 067001 (2003).

32. Note that $n(\mathbf{k})$ will depend on the choice of these $Q_{p}$ because there will be dips for the unoccupied k's.

33. C.T. Shih, Y.C. Chen, C.P. Chou, and T.K. Lee, Phys. Rev. B70, 220502(R) (2004).

34. C.T. Shih, T.K. Lee, R. Eder, C.Y. Mou, and Y.C. Chen, Phys. Rev. Lett. 92, 227002 (2004).

35. T. Tohyama and S. Maekawa, Phys. Rev. B49, 3596 (1994)

36. R.J. Gooding, K.J.E. Vos, and P.W. Leung, Phys. Rev. B49, 4119 (1994).

37. H. Yamase and H. Kohno, Phys. Rev. B69, 104526 (2004).

38. J. Burgy, M. Mayr, V. Martin-Mayor, A. Moreo, and E. Dagotto, Phys. Rev. Lett. 87, 277202 (2001).

39. A. Himeda, T. Kato, and M. Ogata, Phys. Rev. Lett. 88, 117001 (2002). 